Applied Physiology, Nutrition, and Metabolism

Canadian Science Publishing Physiologie appliquée, nutrition et métabolisme

\title{
Perceptions of Exercise Screening Among Older Adults
}

\begin{tabular}{|r|l|}
\hline Journal: & Applied Physiology, Nutrition, and Metabolism \\
\hline Manuscript ID & apnm-2017-0488.R1 \\
\hline Manuscript Type: & Article \\
\hline Date Submitted by the Author: & $28-$ Sep-2017 \\
\hline Complete List of Authors: & $\begin{array}{l}\text { Stathokostas, Liza ; University of Western Ontario, 3M Centre, School of } \\
\text { Kinesiology } \\
\text { Petrella, Andrea; Western University, Kinesiology } \\
\text { Blunt , Wendy; The University of Western Ontario, Western Centre for } \\
\text { Public Health \& Family Medicine (2nd Floor) } \\
\text { Petrella, Robert; The University of Western Ontario, }\end{array}$ \\
\hline $\begin{array}{r}\text { Keyword: } \\
\text { Is the invited manuscript for } \\
\text { consideration in a Special } \\
\text { Issue? : }\end{array}$ & Older adults, Qualitative analysis, Stress test, Physical activity screening \\
\hline
\end{tabular}


Liza Stathokostas, PhD

4 Faculty of Health Sciences, School of Kinesiology, Western University

5 London, ON, N6A 3K7

6 Istatho2@uwo.ca

7

8 Andrea F. M. Petrella, MSc Candidate

9 Faculty of Health Sciences, School of Kinesiology, Western University

10 London, ON, N6A 3K7

11 apetrel4@uwo.ca

12

13 Wendy Blunt, MPH

14 Centre for Studies in Family Medicine, Department of Family Medicine, Western University

15 London, ON, N6G 2M1

16 wblunt@uwo.ca

17

18 Corresponding Author:

19 Robert J. Petrella, MD, PhD

20 Centre for Studies in Family Medicine, Department of Family Medicine, Western University

21 Faculty of Health Sciences, School of Kinesiology, Western University

22 London, ON, N6A 2B7

$23 \quad 519-661-2037$

24 petrella@uwo.ca

25

26 


\section{Abstract}

30 Background: Pre-physical activity screening is important for older adults' participating in physical activity.

31 Unfortunately, many older adults face barriers to exercise participation and thus, may not complete proper

32 physical activity screening. The purpose of this project was to conduct a thematic analysis of perceptions

33 and experiences of community-dwelling older adults regarding pre-physical activity screening (i.e., Get

34 Active Questionnaire (GAQ) and a standardized exercise stress test). Methods: A convenience sample of

35 adults (male $n=58$, female $n=54$ ) aged $75 \pm 7$ years living in the City of London, Ontario was used.

36 Participants completed a treadmill stress test and the GAQ at a research laboratory for community-based

37 referrals. One week later, participants completed the GAQ again and were asked questions by a research

38 assistant about their perceptions of the screening process. Results: Thematic analysis of the responses was

39 conducted. The results indicated that older adults view physical activity screening as acceptable, but not

40 always necessary. Also, the experiences expressed by this sample of older adults indicated that physical

41 activity screening can contribute to continued confidence (through reassurance) and can contribute to

42 increased motivation (through yearly fitness results) in exercise participation. Conclusions: Older adults may

43 perceive screening as supportive in exercise adoption, if screening is simple, convenient and supports older

44 adults' motivation and confidence to exercise. 
52

\section{Introduction}

Current physical activity screening recommendations indicate that the majority of older-aged adults can safely participate in light to moderate physical activities. However, traditionally it was standard for an individual over the age of 65 years to undergo screening prior to beginning a physical activity program and it is still frequently recommended that screening of some type (usually medical in nature and including a stress test) is required for older adults (Resnick et al., 2008). Interestingly, the American College of Sports Medicine and the Centers for Disease Control and Prevention have both suggested that exercise screening should be de-medicalized (Statistics Canada, 2015). As such, there persists a general perception (among the medical community, fitness professionals, and older adults' themselves) of high risk for an adverse event with exercise as we age (Resnick et al., 2008). As health promoters seek to identify and address barriers to promoting physical activity and mobilize the largely inactive older adult population (Health Reports, 2015), the issue of the extent to which physical activity screening tools serve as a facilitator or barrier to exercising have been identified (Resnick et al., 2008). For example, it has been suggested that the routine practice of a pre-physical activity stress test might serve as a barrier to increasing physical activity due to time and cost issues (Gill et al., 2000). The literature in this area is limited, but in a focus group study of older adults who had undergone various forms of physical activity screening, Resnick et al., 2005 found that screening covered a range of different experiences (barriers to motivators) and their perceptions or interpretations of what the screening information was used for also varied. Therefore, the identification that physical activity screening is influenced by each individual's personal situation warrants further investigation to operationalize and tailor screening for older adults. Thus, to inform and optimize the physical activity screening process for the older adult population, the purpose of this study was to obtain the perspectives of older adults undergoing commonly recommended, gold-standard physical activity screening (treadmill stress test) combined with the newly developed Get Active Questionnaire (GAQ) screening questionnaire.

\section{Materials \& Methods}


All of the experimental procedures were approved by the University of Western Ontario Ethics

79 Committee for Research on Human Subjects (Protocol \#108058). All volunteers gave written, informed

80 consent prior to participation in the study.

81

82

\section{Setting}

Data collection, administration of the GAQ, and stress testing took take place at the research laboratory at the Western Centre for Public Health and Family Medicine, Western University, London, Ontario. The laboratory routinely conducts physical activity screening for community-based referrals.

\section{Sample}

A convenience sample of adults (male $n=58$, female $n=54$ ) aged $75 \pm 7$ years living independently in the City of London, Ontario, was used. All individuals greater than 50 years old presenting to the lab for either an annual exercise program stress test or prior to starting an exercise program at the Canadian Center for Activity and Aging or the Retirement Research Association at Western University were eligible for the study. Recruitment was conducted by the stress test assistant/research assistant. Exclusion criteria included a score less than 24 on the Mini Mental State Examination (MMSE; Folstein et al., 1975) or those living in institutional settings, defined as nursing homes or chronic care facilities.

$$
\text { Data collection took place over a seven-month period (June - December 2016). Participants were }
$$
asked to participate in two sessions. On the first day, participants self-completed the GAQ in the presence of the stress test technician/research assistant and then underwent a stress test. The first day session was estimated to take approximately one hour. Seven days later, participants returned to the laboratory. During study visit two (completion of the self-completed GAQ a second time), participants were asked a series of questions to assist the research team in better understanding their perception of the entire physical activity 
screening process (i.e. stress test and questionnaire). One week was determined to be of sufficient duration

102 whereby participants were unlikely to recall their previous responses and there was a low risk of additional

103 adverse event occurrence. The second day session took approximately half an hour to complete.

\section{Stress Test Assessment}

Participants underwent pre-stress test assessments for self-reported health status, anthropometrics, resting blood pressure, cognition (Mini-Mental Status Examination [MMSE] and Montreal

108 Cognitive Assessment [MoCA; Nasreddine et al., 2005]) and recent self-reported health status (i.e., diagnosed conditions, medication use, etc.). Participants then completed a graded-exercise treadmill test. Post-stress test, participants were given the results (and compared to age-matched normative data) and provided a training heart rate to guide their exercise intensity during their exercise program.

\section{Get Active Questionnaire}

The GAQ is a two-page document consisting of four questions on the first page with direction to assess current physical activity and advice to proceed on page two. A second, 'Reference Document' is intended to provide advice on how to proceed when a 'Yes' response is answered on the questionnaire. The GAQ was developed by a committee of exercise professionals and academics and was refined through a

118 Canadian Society for Exercise Physiology (CSEP) member survey. The GAQ was developed to be

119 administered via paper version by the general public (all ages) independently, and/or administered by 120 qualified exercise professionals and health care providers (http://www.csep.ca/home). 
124 assistant. A modified version of questions (Table 1) that has been previously developed for the use in

125 physical activity screening of older adults (Resnick et al., 2005) was utilized. The interview questions were

126 administered to participants verbally and participants wrote their responses. In some cases, the stress test

127 technician would transcribe verbatim when participants were unable to write (i.e., vision problems,

128 tremors, etc.). Guided by the principles of grounded theory (Corbin and Strauss, 1990), content analysis

129 was conducted; an inductive process of coding raw data into overarching themes exploring the subjective

130 experiences of each older adult throughout the GAQ screening process and maximal stress test (Kondracki

131 et al., 2002). Participant responses to questions were reviewed and themes specific to each question were

132 identified by three investigators independently (AP, WB, LS). Two investigators (WB, LS) were not involved

133 with the interview process, and the third investigator was the stress test technician and interviewer (AP).

134 Subsequently, the three investigators met to discuss and finalize the coding of themes and sub-themes

135 related to the older adults' experiences completing the GAQ. Due to the large sample of participants

136 interviewed $(n=100)$, the frequency of common responses by participants were summarized in tables to

137 adequately capture the presence of different themes and sub-themes and draw more meaningful

138 conclusions from the qualitative data (Sandelowski, 2001). Two of the investigators have a background in

139 physical activity screening for older adults $(A P, L S)$ and one of the investigators had expertise in qualitative 140 analysis (WB).

142 Results

\section{Subject Characteristics}

144 Of the 119 individuals presenting to the lab, 113 agreed to participate. None of the participants

145 were excluded to participate based on the exclusion criteria. Both study sessions were completed by 112 of 
146 the participants ( $99 \%$ completion rate). One participant did not complete the GAQ due to time constraints.

147 Participant characteristics are presented in Table 2.

Participant Reponses: Physician or Healthcare Provider Prior to Starting a Physical Activity Program

Responses related to the first question posed (regarding how older adults felt about being evaluated by a physician or health care provider prior to starting an exercise program) yielded two major themes: the need for physical activity screening; and the perception of physical activity screening (Table 3). With respect to older adults who expressed a 'need for physical activity screening', approximately half of the participants reported physical activity screening by a health or exercise professional was necessary, whereas the other half reported screening was not necessary prior to physical activity engagement. Notably, only one subject specifically referred to a preference of being screened by a qualified exercise professional. Of those reporting that screening was necessary, the most frequent response was that it was useful as a precaution. exercise program" some older adults viewed their annual check-ups with their doctor as screening or an equivalent, while other participants stated they would start or change their activity on their own, or viewed the process as not necessary because they were already active. 


\section{Participant Responses: Perceptions of Physical Activity Screening}

175 For those who responded to the question of their 'perception of screening', three sub-themes

176 emerged: that screening was a good idea and they felt comfortable with it; screening was helpful or

177 beneficial; and feeling uneasy about screening. The overwhelming common response by older adults was

178 that they [simply worded] felt "good" and/or are "comfortable" about the screening process. In contrast,

179 there was only one response where an older adult felt uneasy or apprehensive about having to undergo

180 physical activity screening. Some older adults perceived the screening process to be helpful to improve

181 fitness, inform their exercise prescription, and to track their fitness progress (versus as a necessary

182 precaution).

"It can be useful if the physician advises about level of exercise that is appropriate"

"Very useful to guide me during exercise program"

The second question posed to the older adult participants sought to capture if they did any self- 

simply that there was "no impact"; that is, they went through the process and then began exercising. Within this theme, a subtheme was identified where the older adults went ahead and started exercising prior to screening. screening] was necessary"

Only a small percentage of older adults specifically stated that they wanted to wait to be screened 213 prior to starting an exercise program. Only one respondent indicated that if it was up to the older adult 214 themselves to arrange screening, they would have likely put off starting to exercise.

215 Participant Responses: General Experiences During Physical Activity Screening

216 The fourth and final question posed to participants was related to their experiences of all or any of 217 the components during the screening process (Table 6). The responses were grouped into four themes with 
218 responses being specific to either the whole physical activity screening process, the stress test alone, the 219 questionnaire alone, or negative perceptions.

222 results of their stress test.

223

\author{
"Stress test was informative" \\ "Reassuring to being monitored while exercising" \\ "Feel more confident after results" \\ "It gave me reassurance that I am fit to be exercising"
} rather than a physical activity screening tool for safe exercise participation.

"It gave me a sense of reassurance and a benchmark to measure my improvement"

"The stress test and target heart rate range was useful"

"Stress test was beneficial to give me a sense of how my fitness compared to 2 years ago and the aged population"

"I look forward to the stress test. To me it's a challenge to try and maintain or improve on the year before. It keeps me motivated" 
Finally, there was a small group of participants who specifically referred to the sensations that they experienced during the stress test, namely of their level of exertion and feeling in their limbs, but those responses were not associated with any future aversion to physical activity screening. longer but overall it was very good" again a sub-theme of increased confidence was identified.

"No, it was not difficult. I thought the whole process was good"

"It was a good idea. It gave more confidence to do the right thing for me" right direction" completing the physical activity screening questionnaire. Similar to the stress test, older adults indicated

261 that completing the questionnaire gave them a sense of assurance. Although there were some

262 inconsistencies in comments related to the stress test, the responses specific to the questionnaire evoked sentiments specific to various health conditions or status. 
"The questionnaire gave [the participant] a sense of assurance and likes that it helps to spot possible issues"

"It was good to know I didn't have any of the conditions that the study is most concerned about"

"Made me feel good that I could answer "no" to all questions. Made me think maybe I should exercise more since 180 mins is not much for a whole week"

"Not difficult and more confident that I could go ahead with an exercise program because I answered "no" to all questions about my health. I think the questionnaire is helpful to someone starting out in deciding an exercise program. It would identify areas of one's health where a medical assessment would be helpful" included that the questionnaire was not of value, apprehension to do the stress test, and the detail provided to the older adult on the physical activity screening process (Table 6).

Discussion

In an effort to identify the role physical activity screening may play in the initiation and continuation of physical activity among older adults, the present study obtained the perceptions of older adults undergoing commonly used pre- and ongoing physical activity screening procedures with a new screening questionnaire (i.e. GAQ). Our study was unique in that it compared standardized questions asked prior to 
adults. The combined physical activity screening process of a stress test and the GAQ was largely well

received, was considered precautionary, and was generally considered a good idea. However, in this sample

292 of relatively healthy, independently living older adults, the perception of screening prior to physical activity

293 participation varied and was not singularly viewed as an absolute requirement or barrier; as perhaps more

294 widely assumed.

The majority of the older adults included in the present study were regular exercisers and many were a part of a community program which includes an annual stress-test (where a training heart rate and comparison to age-normative values are provided). As such, it is not surprising that some of the older adults perceived the physical activity screening to be more important or to have more value as a fitness measure, versus as a safety measure. A more diverse group of older adults who were questioned on their experiences of having undergone a variety of different screening requirements (not necessarily including a stress test) reported similar results in terms of the necessity of physical activity screening (Resnick et al., 2005). The observations from these two study samples may indicate that non-clinical populations undergoing routine screening do not necessarily share the medical communities' view that the purpose of physical activity screening has been to identify high risk individuals and to avoid adverse events; usually cardiovascular in nature. Moreover, the responses by older adults already physically active and/or those who perceived themselves to be healthy and fit were associated with subthemes indicating that physical activity screening

308 is not necessary for themselves or that they would not hesitate to start on their own. While the participants 309 of the present study reported having various health conditions, numerous studies have identified health 310 problems as a perceived barrier to initiating physical activity participation among older adults ( $\mathrm{O}^{\prime} \mathrm{Neill}$ and 311 Reid, 1991; Bethancourt et al., 2014; Patel et al., 2013; Newson and Kemps, 2007; Kosteli et al., 2016).

312 Therefore, it cannot be ruled out that the need for physical activity screening in the context of safety may 313 be perceived as being more important in clinical populations (Greenwood-Hickman et al., 2016; Desveaux et 314 al., 2016; Simmonds et al., 2016). 
The subtheme of the role of regular or yearly contact with their primary care physician also

317 appeared to influence the respondents' perception of need for physical activity screening; that is, older

318 adults may think that if they are not specifically told they cannot do an activity, this can be considered a 'go-

319 ahead' to participate in physical activity and that further screening is not necessary. In addition, some

320 participants indicated that they did not see the need to hold off participating in physical activity for

321 screening. These observations align with updated recommendations for physical activity pre-participation

322 health screening (applicable to all age-groups); where healthy asymptomatic individuals can initiate light- to

323 moderate-intensity physical activity without physician clearance and those with signs or symptoms and/or

324 known cardiovascular, metabolic, or renal disease or those who intend to engage in vigorous exercise

325 should seek clearance (Riebe et al., 2015). It should be noted, however, that a subset of older adults has

326 been identified who still perceive exercise as potentially harmful (Franco et al., 2015). As such there is still a

327 need for the promotion of awareness of safety related to physical activity in older adults and more

328 expanded role for fitness professionals and physicians to utilize physical activity screening to minimize any

329 fears that may be a barrier to starting exercise.

The concept of 'self-screening' was, not surprisingly, an unfamiliar one to this sample and their

332 interpretation of the question varied. Most of the sample did not do any kind of self-screen and only one

333 person actually referred to a physical activity screening tool, the Physical Activity Readiness Questionnaire

334 (Goodman et al., 2016). As such, there is an opportunity to more widely promote physical activity self-

335 screening and available resources prior to physical activity participation or when significantly changing one's

336 physical activity routine. These results may indicate that there is a role for an easily accessible self-

337 administered physical activity screening tool. The GAQ used in the present study does appear to have a

338 unique role in screening, as it assists the user to identify symptoms or health conditions for which further 
339 consideration or consultation is required before becoming physically active. This was identified by the older

340 adult sample to be specific to the questionnaire and related to increased confidence or reassurance.

341 The process of making an appointment and undergoing the stress test was not viewed as

342 inconvenient or an unpleasant experience by the present sample and thus did not seem to present any kind

343 of barrier. In the United States, the time and cost of seeing a health care provider has been reported to be a

344 burden (Resnick et al., 2005) and concern has been raised specifically with policies which mandate a stress

345 test (Gill et al., 2000). Interestingly, a U.S. sample who participated in focus groups on this topic did not

346 reflect this but identified that exclusion to participating in physical activity as a result of screening was the

347 barrier, as they were frustrated in not being able to begin a program (Resnick et al., 2005). It is unknown

348 whether the older adult population considers the stress test and a physical examination or doctor's

349 permission as gold-standards for pre-physical activity screening, however responses were indicative that the 350 questionnaire was viewed as valid [to the screening process]. These qualitative findings do indicate that the 351 GAQ could be perceived by older adults as an adequate stand-alone physical activity screening tool with the 352 additional benefit of identifying pertinent symptoms and health conditions and may better facilitate the 353 initiation of physical activity.

A consistent facilitator and motivator to engaging in physical activity in the older adult population is to maintain health benefits (Goethe and Kendall, 2016; Schmidt et al., 2016; Trujillo et al., 2004). Improving physical function and maintaining mobility have also been identified as reasons for long-term continuation 358 of a physically active lifestyle (Goethe and Kendall, 2016). Similarly, the annual reassurance of physical 359 fitness status was identified as a positive and confidence building experience by participants in the present 360 study. Hence regular follow-up screening may play a dual role: safe physical activity participation in 361 identifying and any new health risks and feedback regarding progress and maintenance of physical fitness.

362 As older adults tend to have more regular contact with their physician and value their advice, physicians 363 have been identified as playing a key role in the counselling of physical activity (Schutzer and Graves, 2004) 
364 and efforts need to continue to support them in this role. There is also an opportunity to educate older 365 adults on self-screening and specifically when medical clearance is needed. While we did not examine the 366 awareness of the role of exercise professionals in physical activity screening, this could be helpful in 367 supporting the concerns that older adults may have regarding safety and validity of assessments and advice.

\section{Limitations}

This study had some limitations. Demographically, participants were largely Caucasian and lived in a city (London, Ontario) which is categorized as a census metropolitan area (population greater than

372 100,000). The sample population was generally fit, as older adults who participated were also members of 373 community exercise groups. Therefore, our findings may not be generalizable to a wider population of older 374 adults who may be less active or are new to a physical activity program. Finally, the literature is generally 375 limited on this topic hence comparisons were not possible in similar populations and settings. Future studies 376 should focus on physical activity screening as a barrier in clinical populations with complex or multiple

377 morbidity and among those who have restricted access (geographically, services available) to health care for 378 physical activity screening.

Recommendations

The version of the GAQ used here was a preliminary version. Information acquired from this study

382 will be used by CSEP to modify this draft version of the GAQ before a final version is released. Ultimately, 383 the GAQ was largely well received by study participants. In regards to the layout of the GAQ, feedback and 384 observations from participants suggest that the colour scheme of the GAQ (i.e., colour and contrast) made 385 the questionnaire difficult for some older adults to read and thus, complete. Attending to the needs of users 386 who may be colour blind or have vision problems should be addressed in subsequent versions of the GAQ to 387 improve the usability of the questionnaire. On page one of the GAQ (i.e., "Prepare to Become More Active" 388 section), participants suggested changing font and the instruction "within the last six months" to be more 
prominent. If the questionnaire is being administered to an individual, it may be helpful to provide a 390 timeline example (i.e., since January of 2017) to give context for the question. Further, at the bottom of 391 page one ("Prepare to Become More Active" section), clarification or simplifying the instructions at the bottom of the page could be of use. These instructions were frequently misunderstood by participants and those who were not required to complete the "Reference Document" often did so. On page two "Assess Your Current Physical Activity", it was suggested that examples or clarification of what was meant by moderate- to vigorous physical activity would be helpful. Also, participants often miscalculated their exercise minutes per week. As such, these values should be checked by a Qualified Exercise Professional or Healthcare Provider, if applicable.

For the second page "Declaration" section, participants expressed that highlighting the "Check all that Apply" instructions could clarify this to users. Although the list of options provided are inclusive, perhaps reducing or simplifying options would increase the GAQ's usability, as the present sample had great difficulty completing this section. This may improve the GAQ's risk assessment abilities. Further, it may be helpful to provide final instructions after the "Declaration" section to encourage users to start physical activity or provide information to assist those seeking further advice. The utility of the GAQ may be enhanced, especially for those with health conditions (i.e., tremors, colour-blindness, blindness, $\mathrm{MCl}$, etc.). Instructions on related and supportive resources for those looking to become more physically active should be available to individuals completing the GAQ or at minimum, direct users to appropriate resources.

Conclusions always deemed necessary. Physical activity screening can contribute to continued confidence and motivation in exercise participation in this population. This qualitative study provides insight from the

412 perspective of the older adult which can inform the ongoing discussion of safe and beneficial physical 413 activity screening procedures. 


\section{Acknowledgements}

416 The authors would like to thank Ashleigh de Cruz, Cassandra Bartol, Dawn Gill and Narlon Cassio Boa Sorte

417 Silva for their assistance throughout this project with data collection and analysis. Additionally, the authors

418 would like to thank the Canadian Society for Exercise Physiology for funding to support this project.

419 The authors report no conflicts of interest associated with this manuscript.

420

421

422

423

424

425

426

427

428

429

430

431

432

433 
Bethancourt, H.J., Rosenberg, D.E., Beatty, T., and Arterburn, D.E. 2014. Barriers to and Facilitators of Physical Activity Program Use Among Older Adults. J. Clin. Med. Res. 12(1-2): 10-20. doi:10.3121/cmr.2013.1171.

Corbin, J., and Strauss, A. 1990. Grounded Theory Research: Procedures, Canons, and Evaluation Criteria. Qual. Sociol. 13: 4-21.

Desveaux, L., Goldstein, R., Mathur, S., and Brooks, D. 2016. Barriers to Physical Activity Following Rehabilitation: Perspectives of Older Adults with Chronic Disease. J. Aging. Phys .Act. 24(2): 223-33.

Franco, M.R., Tong, A., Howard, K., Sherrington, C., Ferreira, P.H., Pinto ,R.Z., et al. 2015. Older people's perspectives on participation in physical activity: a systematic review and thematic synthesis of qualitative literature. Br. J. Sports. Med. 49: 1268-76. doi:10.1136/bjsports-2014-094015.

Folstein, M.F., Folstein, S.E., and McHugh, P.R. 1975. "Mini-Mental State." A practical method for grading the cognitive state of patients for the clinician. J. Psychiatr. Res. 12: 189-198.

Gill, T.M., DiPietro, L., and Krumholz, H.M. 2000. Role of exercise stress testing and safety monitoring for Healthy Adults and Relevance for Prevention of Cardiovascular Events. Can. J. Cardiol. 32(4): 523-32. Female African American Older Adults. Gerontol. Ger. Med. 2. doi:10.1177/2333721416677399. 
sedentary behavior among overweight and obese older adults. Gerontologist, 56: 660-8.

457

458

459

460

461

462

463

464

465

466

467

468

469

470

471

472

473

474

475

476

Kondracki, N.L., Wellman, N.S. and Amundson, D.R. 2002. Content Analysis: Review of Methods and Their Applications in Nutrition Education. J. Nutr. Educ. Behav. 34: 224-30.

Kosteli, M.C., Williams, S.E., and Cumming, J. 2016. Investigating the psychosocial determinants of physical activity in older adults: A qualitative approach. Psychol. Health. 31(6): 730-49. doi:10.1080/08870446.2016.1143943.

Nasreddine, Z.S., Phillips, N.A., Bedirian, V., Charbonneau, S., Whitehead, V., Collin I., et al. 2005. The Montreal cognitive assessment, MoCA: a brief screening tool for mild cognitive impairment. J. Am. Geriatr. Soc. 53(4): 695-9. doi: 10.1111/j.1532-5415.2005.53221.x.

Newson, R.S., and Kemps, E.B. 2007. Factors That Promote and Prevent Exercise Engagement in Older Adults. J. Aging. Health. 19: 470-81. doi:10.1177/0898264307300169.

O'Neill, K., and Reid, G. 1991. Perceived barriers to physical activity by older adults. Can. J. Public. Health. 82: $392-6$.

Patel, A., Schofield, G.M., Kolt, G.S., and Keogh, J.W.L. 2013. Perceived barriers, benefits, and motives for physical activity: Two primary-care physical activity prescription programs. J. Aging. Phys. Act. 21(1): 85-99.

Resnick, B., Ory, M., Coday, M., and Riebe, D. 2005. Older adults' perspectives on screening prior to initiating an exercise program. Prev. Sci. 6(3): 203-11.

Resnick, B., Ory, M., Coday, M., and Riebe, D. 2008. Professional perspectives on physical activity screening practices: Shifting the paradigm. Crit. Public. Health. 18(1): 21-32.

doi:10.1080/09581590701771717. 
477 Riebe, D., Franklin, B. A., Thompson, P. D., Garber, C. E., and Whitfield, G. P. 2015. Updating ACSM's

478 recommendations for exercise pre-participation health screening. Med. Sci. Sports. Exerc. 47(11):

479 2473-2479. doi:10.1249/MSS.0000000000000664

480 481 484

Get Active Questionnaire found at http://www.csep.ca/home Res. Nurs. Health. 24: 230-40. doi:10.3402/ghw.v11.32914. Med. 39(5): 1056-61. Osteoporosis. Int. 27(3): 979-87. doi: 10.1007/s00198-015-3376-7. 22(4): 348-67. doi:10.1007/s12144-004-1040-z.

Sandelowski, M. 2001. Real qualitative researchers do not count: the use of numbers in qualitative research.

Schmidt, L., Rempel, G., Murray, T.C., Mchugh, T., and Vallance, J.K. 2016. Exploring beliefs around physical activity among older adults in rural Canada. Int. J. Qual. Stud. Health. Well-being. 11:1-8.

Schutzer, K.A., and Graves, B.S. 2004. Review: Barriers and motivations to exercise in older adults. Prev.

Simmonds, B.A.J., Hannam, K.J., Fox, K.R., and Tobias, J.H. 2016. An exploration of barriers and facilitators to older adults' participation in higher impact physical activity and bone health: a qualitative study.

Statistics Canada. 2015. Directly measured physical activity of adults, 2012 and 2013. Retrieved from http://www.statcan.gc.ca/pub/82-625-x/2015001/article/14135-eng.htm

Trujillo, K.M., Brougham, R.R., and Walsh, D.A. 2004. Age differences in reasons for exercising. Curr. Psychol. 
Tables

Table 1. Interview Questions

1. Tell me how you feel about being evaluated by your physician/health care provider/exercise specialist prior to starting an exercise program.

2. Tell me about any self-screening you did prior to starting an exercise program.

3. Tell me how this impacted your willingness to start an exercise program.

4. Tell us any experiences you had in the course of screening. Was it difficult? Did it make you feel more confident about exercise or gave you a sense of assurance? Less confident? Or is screening an inconvenience? If yes, which part (questionnaire, medical exam, stress test)? 
Table 2. Subject Characteristics

\begin{tabular}{|c|c|c|}
\hline Variables & $\mathbf{n}$ & Mean \pm SD \\
\hline \multicolumn{3}{|l|}{ Sex } \\
\hline Male & $58(52 \%)$ & \\
\hline Female & $54(48 \%)$ & \\
\hline Age & & $75 \pm 7$ years \\
\hline $50-54$ & 0 & \\
\hline $55-59$ & $2(2 \%)$ & \\
\hline $60-64$ & $4(4 \%)$ & \\
\hline $65-69$ & $15(13 \%)$ & \\
\hline $70-74$ & $36(32 \%)$ & \\
\hline $75-79$ & $27(24 \%)$ & \\
\hline $80-84$ & $16(14 \%)$ & \\
\hline $85-89$ & $8(7 \%)$ & \\
\hline $90+$ & $4(4 \%)$ & \\
\hline BMI & 111 & $26.5 \pm 3.9 \mathrm{~kg} / \mathrm{m}^{2}$ \\
\hline \multicolumn{3}{|l|}{ Currently Exercising (Self-report) } \\
\hline Yes & $105(94 \%)$ & \\
\hline No & $6(6 \%)$ & \\
\hline Current Smoker & 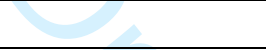 & \\
\hline Yes & $6(6 \%)$ & \\
\hline No & $99(94 \%)$ & \\
\hline \multicolumn{3}{|l|}{ Self-Reported Health Conditions } \\
\hline \multicolumn{3}{|l|}{ High Cholesterol } \\
\hline Yes & $49(44 \%)$ & \\
\hline No & $62(56 \%)$ & \\
\hline \multicolumn{3}{|l|}{ High Blood Pressure } \\
\hline Yes & $42(38 \%)$ & \\
\hline No & $69(62 \%)$ & \\
\hline \multicolumn{3}{|l|}{ Heart Problems } \\
\hline Yes & $7(6 \%)$ & \\
\hline No & 103 (94\%) & \\
\hline \multicolumn{3}{|l|}{ Type II Diabetes } \\
\hline Yes & $8(7 \%)$ & \\
\hline No & 103 (93\%) & \\
\hline \multicolumn{3}{|l|}{ Lung Problems } \\
\hline Yes & $11(10 \%)$ & \\
\hline No & $100(90 \%)$ & \\
\hline \multicolumn{3}{|l|}{ Joint Problems } \\
\hline Yes & $39(35 \%)$ & \\
\hline No & $71(65 \%)$ & \\
\hline $\operatorname{MoCA}(/ 30)$ & 98 & $26 \pm 3$ \\
\hline MMSE (/30) & 100 & $29 \pm 1$ \\
\hline
\end{tabular}

$503 \quad$ MoCA $=$ Montreal Cognitive Assessment. MMSE = Mini-Mental Status Examination. 
Table 3. Themes and subthemes identified from responses to the question "Tell me how you feel about program".

\begin{tabular}{|c|c|c|}
\hline THEME & SUB-THEME & $\mathbf{n}$ \\
\hline \multicolumn{3}{|l|}{ NEED FOR SCREENING } \\
\hline \multirow[t]{3}{*}{ Necessary } & Necessary to consult doctor & 4 \\
\hline & Necessary if starting/changing exercise activity & 2 \\
\hline & Useful precaution & 11 \\
\hline \multirow[t]{4}{*}{ Not Necessary } & Have regular check-ups anyways & 7 \\
\hline & Not necessary & 3 \\
\hline & Not necessary, would start on own or change on own & 4 \\
\hline & Not necessary, already active & 5 \\
\hline \multicolumn{3}{|l|}{ PERCEPTION OF SCREENING } \\
\hline \multirow[t]{2}{*}{ Good } & Good idea & 7 \\
\hline & Good, ok, fine, or comfortable with it & 47 \\
\hline \multirow[t]{4}{*}{ Helpful/Beneficial } & Helpful to improve fitness & 1 \\
\hline & Helpful to guide exercise prescription & 2 \\
\hline & Beneficial information on physical condition & 3 \\
\hline & Beneficial information to track progress & 3 \\
\hline \multirow[t]{2}{*}{ Uneasy } & Uneasy about process/results & 1 \\
\hline & Total: & 100 \\
\hline
\end{tabular}

508 
510 Table 4. Themes and subthemes identified from responses to the question "Tell me about any self-

511 screening you did prior to starting an exercise program".

\begin{tabular}{|l|l|c|}
\hline THEME & SUB-THEME & $\mathbf{n}$ \\
\hline & Yes, necessary for self, reassuring, good idea & 3 \\
\hline & Yes, checked with doctor & 4 \\
\hline & No self-screening & 66 \\
\hline & Not self-screening, annual check-up & 15 \\
\hline & Not necessary, already active & 10 \\
\hline & Monitor on daily basis and/or prior to any exercise & \\
\hline & activity (often associated with a chronic condition) & 100 \\
\hline & & Total: \\
\hline
\end{tabular}

512

513

514

515

516

517

518 
521 Table 5. Themes and subthemes identified from responses to the question "Tell me how screening

522 impacted your willingness to start an exercise program. Did you put off starting because you would have to

523 make an appointment to see a doctor?"

\begin{tabular}{|l|l|c|}
\hline THEME & SUB-THEME & $\mathbf{n}$ \\
\hline No Impact & No impact & 68 \\
\hline & No impact, went ahead with exercise & 9 \\
\hline Delayed Starting & No impact, there is no need to consult doctor to start & 7 \\
\hline Potential Delay & Wanted to get screened before starting & 4 \\
& If onus was up to subject to arrange appointment, would & 1 \\
\hline & probably put off. & \\
\hline & & Total: \\
\hline
\end{tabular}

524

525 
533 Table 6. Themes and subthemes identified from responses to the question "Tell us any experiences you had

534 in the course of screening. Was it difficult? Did it make you feel more confident about exercise or gave you

535 a sense of assurance? Less confident? Or is screening an inconvenience? If yes, which part (questionnaire,

536 medical exam, stress test)?"

\begin{tabular}{|c|c|c|}
\hline THEME & SUB-THEME & \# \\
\hline \multirow[t]{3}{*}{ Stress Test } & Stress test increased confidence and/or was reassuring & 26 \\
\hline & $\begin{array}{l}\text { Stress test results and training heart rate provided good } \\
\text { to gauge fitness and improve fitness }\end{array}$ & 17 \\
\hline & Stress test was physically challenging & 6 \\
\hline \multirow[t]{4}{*}{ Whole Screening Process } & Whole process was ok, felt good about it & 8 \\
\hline & $\begin{array}{l}\text { Whole process (questionnaire and stress test) increased } \\
\text { confidence }\end{array}$ & 6 \\
\hline & Screening reinforced positive attitude towards exercise & 2 \\
\hline & Process was not inconvenient & 17 \\
\hline \multirow[t]{3}{*}{ Questionnaire } & Questionnaire was reassuring & 7 \\
\hline & No problem with questionnaire, easy to figure out & 15 \\
\hline & Questionnaire was confusing & 3 \\
\hline \multirow[t]{4}{*}{ Negative Perceptions } & Screening not relevant/no value & 2 \\
\hline & Apprehensive to do stress test & 2 \\
\hline & Would like more detailed pre-test instructions & 1 \\
\hline & $\begin{array}{l}\text { Questionnaire did not provide enough info to address } \\
\text { health concerns }\end{array}$ & 1 \\
\hline
\end{tabular}

